\title{
Pouring fuel on the fire: Th17 cells, the environment, and autoimmunity
}

\author{
Patrick R. Burkett, ${ }^{1,2}$ Gerd Meyer zu Horste, ${ }^{1}$ and Vijay K. Kuchroo ${ }^{1}$
}

'Evergrande Center for Immunologic Diseases, Harvard Medical School and Brigham and Women's Hospital, Boston, Massachusetts, USA. ²Pulmonary and Critical Care Division, Department of Medicine, Brigham and Women's Hospital, Boston, Massachusetts, USA

\begin{abstract}
Cytokines play a critical role in controlling the differentiation of CD4 Th cells into distinct subsets, including IL-17producing Th17 cells. Unfortunately, the incidence of a number of autoimmune diseases, particularly those in which the IL23/IL-17 axis has been implicated, has risen in the last several decades, suggesting that environmental factors can promote autoimmunity. Here we review the role of cytokines in Th17 differentiation, particularly the role of IL-23 in promoting the differentiation of a pathogenic subset of Th17 cells that potently induce autoimmune tissue inflammation. Moreover, we highlight emerging data that indicate that environmental factors, including the intestinal microbiota and changes in diet, can alter normal cytokine regulation with potent effects on Th17 differentiation and thus promote autoimmunity, which has strong implications for human disease.
\end{abstract}

\section{Introduction}

The random rearrangement of adaptive antigen receptors allows for incredible diversity of antigen receptor specificity but comes at the cost of creating potentially autoreactive T and B lymphocytes. Although many potentially autoreactive clones are deleted during development or are prevented from causing disease by the various mechanisms of peripheral tolerance, others go on to induce autoimmune disease. Unfortunately, the incidence of autoimmune diseases such as MS, type 1 diabetes (T1D), and inflammatory bowel disease (IBD) has been increasing in the last three to four decades (1-3). Thus it is more important than ever to understand the pathways that promote the differentiation and effector function of self-reactive CD4 Th cells into pathologic effector subsets.

One of the principal means by which Th cells coordinate immune responses is by producing cytokines. Over 20 years ago, Mosmann and Coffman recognized that Th cells can be divided into distinct subsets on the basis of unique patterns of cytokine production (4). While many different subsets have been identified in the ensuing 20 years, the most well-defined effector Th subsets include Th1 cells, which make IFN- $\gamma$; Th2 cells, which produce IL-4; and Th17 cells, which generate IL-17 (Figure 1). The patterns of cytokine production of these subsets endow them with different functional properties, and these subsets therefore clear different types of pathogens by inducing distinct types of inflammation. Cytokines also play critical roles in regulating the differentiation of naive Th cells into different effector subsets, independently of antigen specificity, by inducing expression of subset-defining transcriptions factors (5). Thus, cytokines control the differentiation of Th cells as well as their effector function.

Given the importance of cytokines in regulating Th cells, it is not surprising that polymorphisms or environmental stimuli that

Conflict of interest: The authors have declared that no conflict of interest exists. Reference information: J Clin Invest. 2015;125(6):2211-2219. doi:10.1172/JCI78085. alter the normal regulation of cytokines are frequently identified as risk factors for the development of autoimmune disease. In particular, mutations or stimuli that result in the dysregulation of cytokines that control Th17 cells appear to particularly predispose to the development of several autoimmune diseases $(6,7)$. In this Review we focus on the role of cytokines in controlling Th17 cell differentiation and effector function and discuss how environmental factors, such as diet and the intestinal microbiota, may predispose to the development of a pathogenic subset of Th17 cells that can promote autoimmune disease.

\section{Cytokine regulation of Th17 differentiation}

The observation that IFN- $\gamma$-producing Th cells are prevalent in many autoimmune diseases, such as MS and RA, led to the hypothesis that Th1 cells were critical for disease pathogenesis. Paradoxically, in mouse models of RA and MS (collagen-induced arthritis or EAE), mutations in key Th1-related genes such as Ifng, Ifngr, and Stat1 lead to increased susceptibility to disease, rather than protection from disease (8-10). Moreover, while mice deficient for the p40 subunit of IL-12 were resistant to EAE, p35 subunit-deficient mice were still susceptible. This apparent conundrum was resolved by the recognition that another subunit, p19, could also pair with $\mathrm{p} 40$ to form the cytokine IL-23 and that mice lacking p19 were resistant to EAE despite having normal Th1 responses (11-13). Rather than promoting Th1 differentiation, as IL-12 does, IL-23 induced IL-17-producing Th cells, later termed Th17 cells, which can induce autoimmune tissue inflammation $(13,14)$. Moreover, IL-23 was also found to be crucial to developing intestinal inflammation in several mouse models of IBD (15-17). Thus, IL-23 plays a vital role in promoting $\mathrm{T}$ cell-mediated tissue inflammation in murine models of human autoimmune disease, in large part by promoting Th17 differentiation.

While IL-23 is clearly critical for the development of Th17 cells in vivo, naive Th cells do not express IL-23 receptor (IL-23R) (18), suggesting that other cytokines are responsible for the initial 


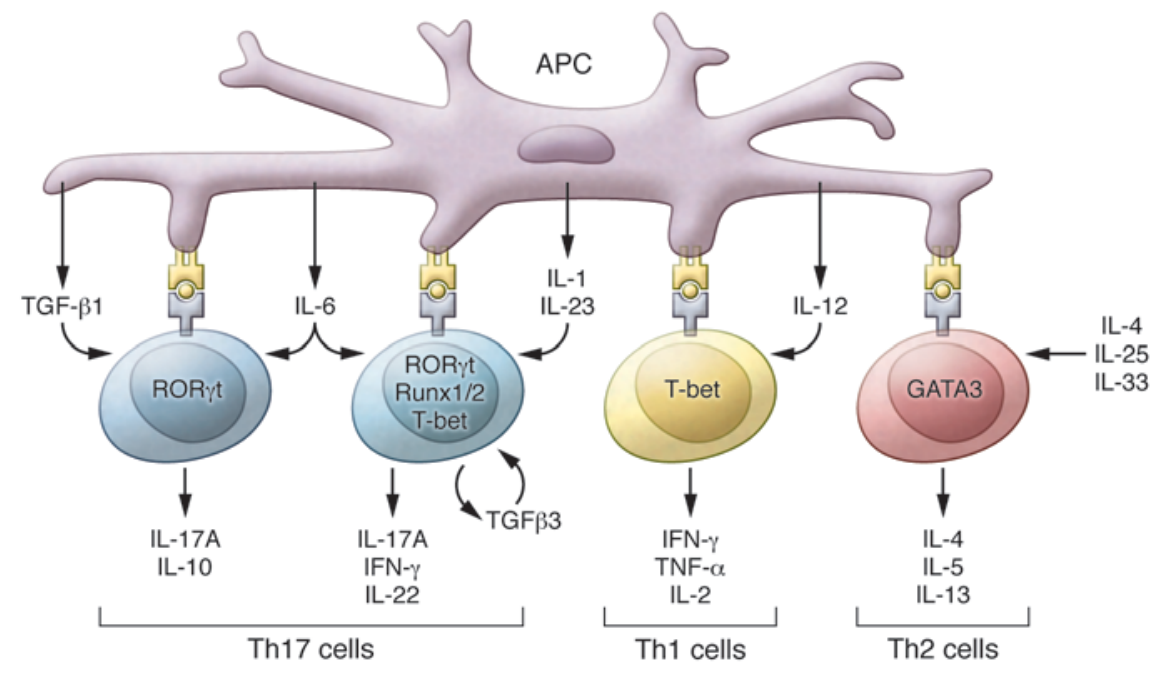

Figure 1. Effector $T$ cell subsets. The subsets of effector Th cells are shown, along with the cytokines that drive their differentiation, lineage-defining transcription factors, and the cytokines they produce. Within the Th17 subset, two distinct subtypes of cells have been described with differing abilities to induce autoimmunity: a pathogenic subtype that produces both IL-17 and IFN- $\gamma$ and a nonpathogenic subtype that produces IL-17 and IL-10. induction of this lineage. The combination of TGF- $\beta 1$ and IL- 6 efficiently induces IL-17-producing murine $\mathrm{T}$ cells in vitro and highlights the reciprocal relationship between induced Tregs, which are generated by TGF- $\beta 1$ alone, and Th17 cells $(19,20)$. A series of studies demonstrated that IL-21, a $\gamma_{\mathrm{c}}$-dependent cytokine, could promote the differentiation of Th17 cells (21-23). In combination with TGF- $\beta$, IL-21 induces IL-17 production from naive Th cells and promotes the expression of both ROR $\gamma \mathrm{t}$, the lineage-defining transcription factor of Th17 cells, and IL-23R $(23,24)$. IL-21 could also inhibit the generation of TGF- $\beta$-induced FOXP $3^{+}$Tregs, and analysis of IL-6-deficient mice revealed that the ability of IL-21 to generate Th17 cells from Th cells was independent of IL-6 (21). Both IL-6 and IL-21 can induce Th17 cells themselves to produce IL-21. This led to a model of Th17 cell differentiation in which IL- 6 elicits autocrine IL-21 production, which then amplifies the Th17 response by inducing itself and upregulating IL-23R, allowing for the stabilization and terminal differentiation of the lineage through IL-23. While IL-21 can clearly promote Th17 differentiation in vitro, it is not absolutely required in vivo. In particular, both IL-21- and IL-21R-deficient mice are not protected from EAE, indicating that IL-21 may have a redundant role in Th17 differentiation in this setting $(25,26)$. In contrast, IL-21 is absolutely required in the NOD model for development of spontaneous diabetes, as loss of either Il21 or Il21r makes mice resistant to the development of disease $(27,28)$. Moreover, SNPs in IL21 and IL21R have been implicated in several human autoimmune diseases (29-34).

Interestingly, Th17 cells differentiated with just TGF- $\beta 1$ and IL-6 are inefficient at inducing autoimmunity when transferred in vivo, in part due to their co-production of IL-10 (35). However, the addition of IL-23 to TGF- $\beta 1$ and IL- 6 induces Th17 cells that trigger EAE after transfer, supporting a key role for IL-23 in promoting the ability of Th17 cells to induce tissue-specific autoimmunity (36). Interestingly, the role of TGF- $\beta 1$ in human Th17 cell differentiation is less clear cut, as studies have shown variable importance of this cytokine for human Th17 cell differentiation (37-39). Moreover, human Th17 cells can be efficiently induced by the combination of IL-1 $\beta$, IL- 6 , and IL-23, and subsequent work suggests that TGF- $\beta 1$-dependent Th17 cells may have different functional properties than those differentiated with $\operatorname{IL}-1 \beta(38,40)$.
Murine Th17 differentiation can also be induced in the absence of TGF- $\beta 1$ signaling, via the combination either of IL- $1 \beta$, IL- 6 , and IL- 23 or of TGF- $\beta 3$ and IL- $6(41,42)$. These TGF- $\beta 1$-independent Th17 cells can efficiently induce autoimmune tissue inflammation upon adoptive transfer, a trait that led to them being termed "pathogenic" Th17 cells. Additionally, they possess a gene expression profile distinct from that of "nonpathogenic" Th17 cells, which are differentiated with TGF- $\beta 1$ and IL-6.

These observations have led to two competing hypotheses. The first posits that IL-23 cements Th17 lineage commitment such that Th17 cells induced with TGF- $\beta 1$ and IL- 6 are insufficiently committed to the lineage and cannot induce autoimmune tissue inflammation. The second suggests that there are distinct subtypes of Th17 cells whose differentiation is dependent on distinct combinations of cytokines. The latter hypothesis is supported by the finding that pathogenic Th17 cells express higher levels of several Th1 lineage-associated transcripts, such as the transcription factor T-bet and IFN- $\gamma$, and have been shown to be derived from IL-17 single producers by fate mapping experiments $(43,44)$. Similarly, an important feature of human Th17 cells, as well as those isolated from inflamed tissues in mouse models of Th17-mediated disease, is that they coproduce IL-17 with other cytokines, leading to distinct functional roles (45-47). For instance, human Th17 cells that produce both IL-10 and IL-17 preferentially respond to Staphylococcus aureus, while those that produce both IL-17 and IFN- $\gamma$ recognize Candida albicans, suggesting that distinct pathogens induce distinct subtypes of Th17 cells (40). IL-17/IFN- $\gamma$ coproducers have been ascribed pathogenic functions in the CNS during EAE; however, it has been difficult to reliably differentiate such cells in vitro. Repeated rounds of in vitro culture in the presence of either Th1-inducing cytokines or IL-23 can induce IL-17/IFN- $\gamma$ coproducers, albeit at moderate percentages. The transcription factors T-bet, RUNX1, and RUNX3 appear to be important in the acquisition of this phenotype $(48,49)$.

Large-scale transcriptional analysis of Th17 differentiation at very high temporal resolution has begun to elucidate the molecular networks that control this process, and is a promising approach to the identification of novel regulatory molecules that control pathogenic Th17 differentiation $(50,51)$. In fact, Th17 development is controlled 


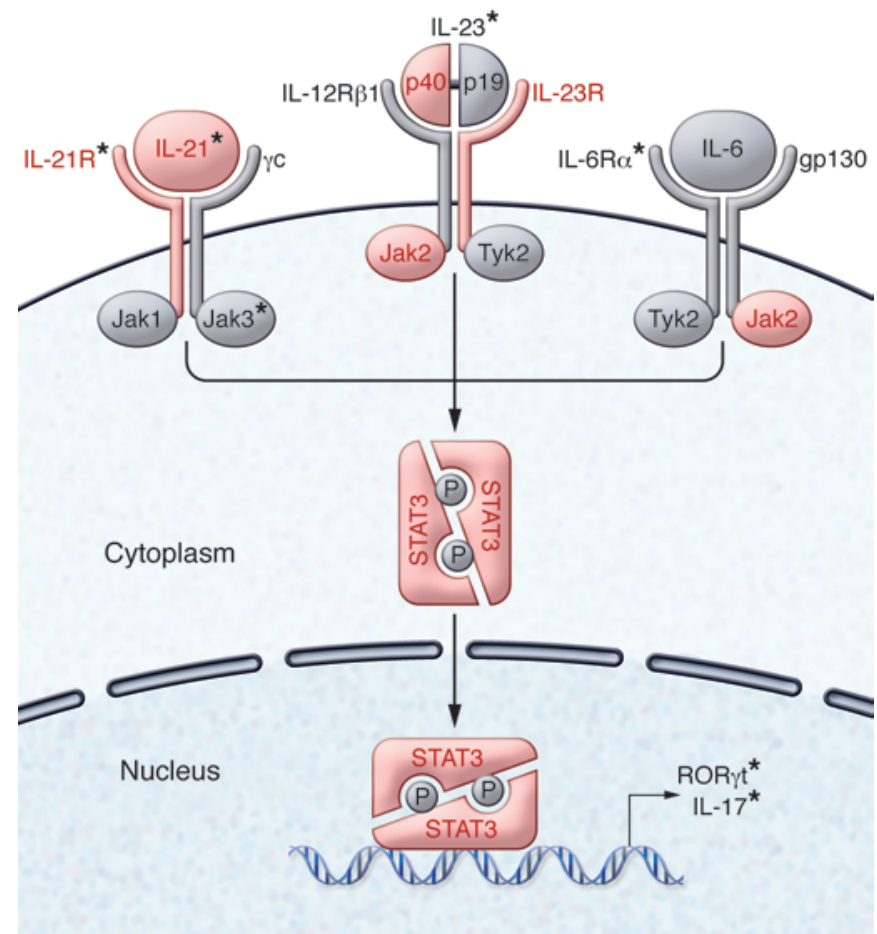

by two self-reinforcing, mutually antagonistic modules: a positive module containing 22 genes that promote Th17 differentiation and a negative module of 5 genes that inhibit Th17 development. These mutually antagonistic modules are essential for maintaining a balance between Th17 cells and other T cell subsets. Thus, a growing amount of evidence suggests that there may be at least two distinct subtypes of IL-17-producing Th cells and that these subtypes may mediate distinct immunologic functions, although the underlying molecular basis for these subtypes is just beginning to be identified.

\section{IL-23 and autoimmunity}

Mice that overexpress IL-23 develop a spontaneous inflammatory disease similar to ankylosing spondylitis, while mice lacking tristetraproline, which regulates I 23 mRNA stability, develop spontaneous systemic inflammation, highlighting the importance of this cytokine in regulating inflammatory T cell populations (52, 53). Moreover, IL-23 is essential for the induction of the pathogenic Th17 cell gene expression profile (41). Thus, it is not surprising that the IL-23R is expressed at higher levels in pathogenic Th17 cells and is required for Th17-mediated induction of EAE (41). The pathways activated downstream of IL-23R that are required for pathogenicity are just beginning to be identified. One such molecule, the salt-sensitive kinase SGK1, was recently identified through use of transcriptional networks and predicted proteinprotein interactions, experimentally verified to be downstream of IL-23R, and shown to be required for the induction of pathogenic Th17 cells $(50,54,55)$. Additionally, the IL-23R-mediated induction of the cytokine GM-CSF is required for the pathogenicity of Th17 cells, although by itself it is not sufficient to induce EAE in vivo $(56,57)$. Thus, while further investigation into the molecular basis for the differentiation of pathogenic Th17 cells is clearly needed, the critical role of IL-23 in this process is evident.
Figure 2. STAT3-dependent cytokines in autoimmunity. Cytokines that activate STAT3, including IL-6, IL-21, and IL-23, play a critical role in promoting Th17 differentiation and have been implicated in human autoimmune disease. The signaling pathways of IL-23, IL-21, and IL- 6 are shown. Genes marked in red have SNPs that have been implicated in human autoimmune diseases. Genes marked with an asterisk have targeted therapeutics either approved or in active clinical development for treatment of autoimmune disease.

The importance of IL-23 in promoting autoimmune tissue inflammation is further highlighted by the finding that polymorphisms in genes related to IL-23 and its signaling pathway are associated with several human autoimmune diseases, including IBD, ankylosing spondylitis, psoriasis, and autoimmune thyroiditis (Figure 2 and refs. 58-60). SNPs in IL23R are associated with both Crohn's disease and ulcerative colitis, consistent with the crucial role IL-23 has in mouse models of $\operatorname{IBD}(15,17,61)$. One IBD-associated IL23R SNP, which encodes the mutation R381Q, lies within exon 9 of IL23R and appears to be protective, with a reduced frequency in patients compared with controls. After activation, $T$ cells isolated from individuals expressing IL-23R ${ }^{\mathrm{Q} 381}$ have a reduced frequency of IL-23R ${ }^{+}$Th cells and decreased phosphorylation of STAT3 after stimulation with IL-23 (62). Moreover, SNPs in the gene encoding IL-12/23p40, which could affect both IL-12 and IL-23 function, have also been linked to psoriasis, psoriatic arthritis, and IBD (63-66). Thus, SNPs affecting the IL-23 pathway are commonly found in human autoimmune disease, and a SNP that results in decreased IL-23R function is protective against the development of intestinal inflammation. These data suggest that the proper regulation of IL-23 is critical to prevent autoimmunity.

STAT3 represents an important downstream mediator of IL23R signaling, as well as several other inflammatory cytokines, including IL-6 and IL-21, and is crucial for Th17 differentiation in both humans and mice. SNPs in STAT3 have been linked to many of the same human autoimmune diseases associated with the IL-23 pathway, including psoriasis, IBD, and ankylosing spondylitis (6668). Loss-of-function mutations in STAT3 lead to the development of autosomal-dominant hyper-IgE syndrome, manifestations of which include chronic mucocutaneous candidiasis and recurrent staphylococcal infections. These patients have markedly decreased frequencies of IL-17- and IL-22-producing T cells (6972). In contrast, several patients have recently been identified who had very early onset multi-organ autoimmune disease and were found to have novel STAT3 mutations that resulted in increased STAT3 activity $(73,74)$. Such patients have a reduced frequency of circulating $\mathrm{FOXP}^{+}$Tregs, and one patient responded clinically to blockade of IL-6R with tocilizumab with a marked decrease in the frequency of IL-17-producing CD4 ${ }^{+} \mathrm{T}$ cells (74). Thus, mutations in STAT3 can alter Th17 differentiation, and STAT3 mutations and polymorphisms can predispose to the development of autoimmune disease, highlighting the importance of this molecule.

\section{Regulation of Th17 differentiation by environmental factors}

The relatively recent increase in the incidence of a variety of autoimmune diseases in which Th17 cells and IL-23 have been implicated, including MS and IBD, suggests that environmental factors 
may lead to altered immune responses and promote autoimmunity. Given the importance of cytokines in regulating Th17 differentiation and the apparently crucial role of IL-23 in promoting a pathogenic Th17 subset, it is important to understand how environmental stimuli can influence regulation of Th17-related cytokines, particularly IL-23. Mucosal tissues such as the intestine have emerged as a key physiologic site for the differentiation and regulation of Th17 cells, suggesting that these cells may be particularly sensitive to perturbations from changes in environmental stimuli.

Microbiota. The intestine is home to both a vast array of commensal microbes and a multitude of immune cells, and the interplay between these two populations has been increasingly recognized to affect immune homeostasis. At steady state, a significant proportion of the Th cells residing in the lamina propria of the small intestine are Th17 cells (24). Colonization of germfree mice with normal intestinal microbiota induces the upregulation of a number of cytokines, including TNF- $\alpha$, IFN- $\gamma$, IL-1, IL- 6 , IL-10, and IL-17; however, the induction of IL-17 in this setting is profound, showing an increase of over 1,000-fold (75-77). Consistent with this, the development of intestinal Th17 cells requires the presence of the intestinal microbiota, as these cells are absent in germ-free mice (76). The intestinal microbiota are also required for the maintenance of intestinal Th17 cells, as antibiotic treatment, particularly antibiotics with activity against Gram-positive organisms, results in marked depletion of this population $(76,77)$. Thus, normal commensal microbes induce an environment that promotes the differentiation of Th17 cells.

Ivanov and colleagues made the observation that mice from Taconic Farms have a substantially higher frequency of IL- $17^{+}$ Th cells in the lamina propria than do mice from The Jackson Laboratory (76). Cohousing of Jackson and Taconic mice led to an increased frequency of lamina propria Th17 cells in Jackson mice, suggesting that the bacteria responsible could be transmitted between mice (76). A single Gram-positive bacterial species, termed segmented filamentous bacteria (SFB), appears to be primarily responsible for inducing intestinal Th17 cells. This Clostridial-related species is non-culturable, sporulating, and strongly adherent to ileal epithelium and is nearly absent in mice from The Jackson Laboratory $(78,79)$. Inoculation of Jackson mice with fecal microflora from SFB-monocolonized mice potently induces expansion of intestinal IL-17 $\mathrm{IL}^{-22^{+}}$Th cells (79). The majority of these cells are specific for SFB-derived antigens, although there are conflicting data regarding whether recognition of cognate antigen is required for their differentiation (80-83). SFB colonization also induces development of secondary and tertiary lymphoid structures in the intestine, and the development of SFB-specific Th17 cells requires such organized structures (84). Thus, a single commensal microbial species is able to exert a marked effect on Th cell differentiation, augmenting the differentiation of a subset important in the pathogenesis of autoimmune disease.

The intestinal microflora, and in particular the presence of SFB, has been demonstrated to alter the course of a number of mouse models of autoimmune disease. In a Th cell transfer model of colitis, which requires the presence of intestinal microbiota, reconstitution of mice with a mixture of SFB and conventional murine microbiota led to colitis, whereas either one alone led to attenuated disease (85). Inflammatory autoimmune arthritis can be mitigated in the $\mathrm{K} / \mathrm{BxN}$ mouse model by treatment with either anti-IL-17 or antibiotics that cover Gram-positive organisms, is significantly attenuated in germ-free mice, and recurs upon either conventionalization or SFB monocolonization (86). A spontaneous model of relapsing/remitting EAE has been found to be dependent on the presence of commensal microbiota (87). Similarly, in germ-free animals, EAE induced by immunization with complete Freund's adjuvant/myelin oligodendrocyte glycoprotein is attenuated compared with EAE in conventionalized mice, and these mice show an increased frequency of Tregs and decreased numbers of $\mathrm{IL}^{-17^{+}} \mathrm{CD} 4^{+}$cells (88). After monocolonization with SFB, germ-free mice are once again susceptible to EAE, with markedly enhanced Th17 responses in both the spinal cord and small intestine. This suggests that the SFB-induced microenvironment can foster the differentiation of autoreactive Th17 cells that can then circulate throughout the body and induce inflammatory disease at distal sites, depending on the specificity of the TCRs of the T cells. This possibility is further supported by the finding that adoptively transferred naive Th cells that express an SFB-specific TCR upregulate ROR $\gamma \mathrm{t}$ in an SFB-dependent manner and can be found in both the intestine and the spleen (81). In contrast, in the case of the NOD model of T1D, SFB colonization actually appears to be protective, as SFB-positive NOD mice have a dramatically reduced incidence of diabetes (89). Thus, SFB-induced intestinal Th17 cells can variably modulate the course of organ-specific autoimmune disease.

The mechanisms by which SFB induce intestinal Th17 cells are still being elucidated. Despite playing a critical role in regulating intestinal epithelial homeostasis, TLR-derived MyD88-dependent signals appear to be dispensable for SFB-induced intestinal Th17 differentiation $(75,76,90)$. However, several studies have suggested an important role for intestinal APCs in promoting the differentiation of microbiota-dependent intestinal Th17 cells via both antigen-dependent and -independent mechanisms (75, 79-81). Intestinal Th17 cells show a substantial degree of reactivity to SFB-derived antigens and fail to develop in the absence

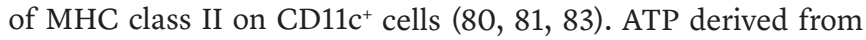
lumenal microbes specifically induces upregulation of IL-23p19, IL-6, and TGF- $\beta$-activating integrins in a $C D 70^{\text {hi }} C D 11 c^{\text {lo }}$ population uniquely present in the lamina propria that can support the differentiation of Th17 cells in vitro (75). Intriguingly, mice lacking Entpd7, which encodes an extracellular ATP hydrolase preferentially expressed on small intestinal epithelium, have both increased luminal ATP and higher frequencies of intestinal Th17 cells (91). Moreover, while Entpd7-deficient mice are resistant to Citrobacter rodentium infection, they develop more severe EAE than WT mice. Thus, microbial-derived peptides and metabolites contribute to the induction of intestinal Th17 cells.

Type 3 innate lymphoid cells (ILC3s), which resemble Th17 cells with regard to the cytokines they produce, appear to regulate intestinal Th17 cell differentiation in a microbiota-dependent manner. Mice lacking Ahr, IL-22, IL-23p19, or IL-23R have relative outgrowth of SFB and increased intestinal Th17 cells, and this appears to be due to a marked decrease in IL-22-producing immune cells, particularly ILC3s (92-94). IL-22 is critical for maintaining intestinal barrier function, and in the absence of IL-22, SFB increase in relative abundance, leading to an increased 


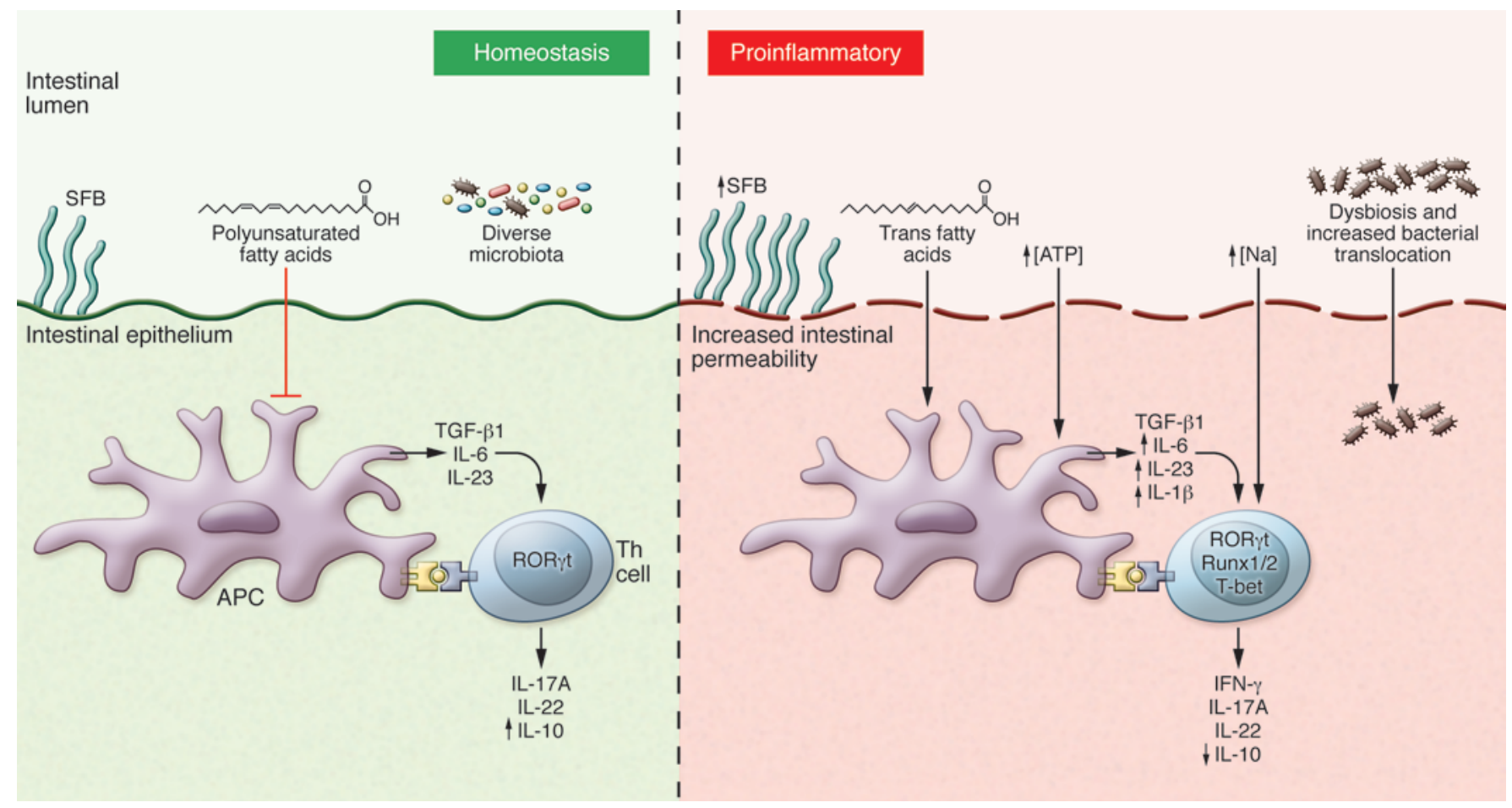

Figure 3. Factors that alter the intestinal microenvironment can affect Th17 differentiation. The small intestine plays an important role in promoting Th17 differentiation in vivo, and changes in dietary intake or the intestinal microbiota can alter cytokine production in that microenvironment and promote the differentiation of Th17 cells with a pathogenic phenotype. This more pro-inflammatory environment may predispose to the development of autoimmune disease. Intriguingly, many of these same factors can also predispose to the development of metabolic diseases such as type 2 diabetes. Thus, dysregulation of Th17-related cytokines may also play a role in the pathobiology of these diseases as well as in full-blown autoimmunity.

frequency of Th17 cells in both the intestine and distal secondary lymphoid tissue $(93,95,96)$. IL-23-induced IL-22 also appears to be critical for prohibiting microbiota from entering normally restricted anatomical compartments, as mice deficient in both IL-23R and RAG2 have elevated levels of LPS in both the spleen and liver (93). Furthermore, selective depletion of ILCs results in systemic dissemination of Alcaligenes spp., a normal enteric commensal (97). Dissemination of Alcaligenes induced a systemic inflammatory response that was prevented by the administration of exogenous IL-22. Intriguingly, pediatric patients with Crohn's disease had higher titers of anti-Alcaligenes antibodies compared with healthy controls, suggesting that dissemination of these bacteria may occur in patients with IBD as well. Finally, ILC3s have also been found to express MHC class II and induce peripheral tolerance to commensal-derived antigens, as selective deletion of the MHC class II locus in ILC3s leads to spontaneous intestinal inflammation associated with expansion of intestinal Th17 cells $(80,92)$. Thus, the interplay between ILC3s and intestinal microbiota can modulate in vivo Th17 differentiation by a number of distinct mechanisms.

Diet. The increased incidence of autoimmune disease over the last several decades has mirrored the widespread adoption of a diet dependent on processed foods rich in salt, fats, and both sugar and artificial sweeteners. While it is currently unclear whether these two things are related, there is a clear association between obesity and the adoption of a diet heavy in processed foods. Recent work has clearly highlighted the extent to which obesity is an inflam- matory state, with increased production of cytokines known to promote Th17 differentiation, including IL-1 and IL-6 (98). Moreover, mice placed on a high-fat diet demonstrate increased expression of IL-17, IL-23p19, and ROR $\gamma$ t consistent with increased Th17 responses and have more rapid onset of colitis (99). Similarly, mice placed on a diet rich in trans fatty acids, which are found in processed hydrogenated vegetable oil, develop more severe intestinal inflammation in response to dextran sodium sulfate, and this is associated with an increase in both IL-17 and ROR $\gamma \mathrm{t}$ expression (100). Trans fatty acids also directly induce increased expression of IL-23p19 in peritoneal macrophages. However, addition of polyunsaturated fatty acids (PUFAs), which have been shown to mitigate obesity-associated metabolic pathology, results in decreased colitis severity in mice placed on a high-fat diet that otherwise would exacerbate disease, and this correlates with decreased levels of a number of Th17-related cytokines (101-103). Interestingly, observational data from the Nurse's Health Study suggest a possible correlation between dietary PUFA intake and a reduced risk of ulcerative colitis, whereas high intake of trans-unsaturated fats was associated with an increased risk of developing ulcerative colitis (104). Thus, both obesity and dietary fat intake can alter the production of cytokines involved in Th17 differentiation and potentially predispose to the development of autoimmunity.

In addition to fats, processed foods are also rich in salt, and recent work has shown that a diet high in salt may promote both Th17 differentiation and induction of autoimmunity $(54,55)$. This appears to be directly mediated by the salt-sensitive kinase, 
SGK1, which was identified as an important downstream target of IL-23R signaling $(50,54)$. Increased salt concentrations preferentially induced Th17 cells with a pathogenic, pro-inflammatory gene signature. Consistent with this, mice placed on a highsalt diet develop more severe EAE $(54,55)$. While a high-salt diet induces multiple physiological changes, it appears that it alters EAE severity by directly affecting $\mathrm{T}$ cell priming, since mice with a T cell-specific deletion of $S g k 1$ have reduced EAE severity and do not develop exacerbated disease on a high-salt diet (54). Given how tightly mammals regulate the plasma sodium concentration, it is tempting to speculate that the high-sodium diet may exert its effect by altering the differentiation of intestinal Th17 cells toward a pathogenic phenotype, since they would be in close proximity to the increased concentration of sodium in the intestinal lumen; however, this remains to be demonstrated. Although there is no conclusive evidence, a number of recent studies support the concept that a high-salt diet may predispose to the development of human autoimmune diseases. One such study found a positive correlation between sodium intake and disease activity in patients with relapsing-remitting MS, as patients with higher salt intake had higher lesion load by MRI and higher disability scores (105). Similarly, the combination of high sodium intake and smoking was associated with a significantly increased incidence of developing RA (106). Thus, high salt concentrations can promote the differentiation of pathogenic Th17 cells, and increased dietary salt intake may promote autoimmune disease in both mice and humans.

\section{The IL-23/IL-17 axis as a therapeutic target in autoimmunity}

The identification of the IL-23/IL-17 axis as critical for human autoimmune disease has led to the rapid development of many novel therapeutic agents. Monoclonal antibodies to a variety of cytokines and cytokine receptors and small-molecule inhibitors of ROR $\gamma \mathrm{t}$ and JAKs are in active preclinical and clinical development (Figure 2). Clinical trials of these agents in human autoimmune diseases suggest that they hold promise as therapeutics and illuminate the distinct roles these cytokines play in different autoimmune diseases.

Humanized monoclonal antibodies to both IL-17 (secukinumab) and IL-17RA (brodalumab) significantly improved moderate to severe psoriasis as well as psoriatic arthritis (107-110). Moreover, a Phase II clinical trial with secukinumab in MS has been completed, and preliminary results have been reported to be positive (ClinicalTrials.gov identifier: NCT01051817). In contrast, a Phase II study on RA patients with inadequate response to methotrexate reported no significant response to secukinumab, while a Phase Ib study of brodalumab failed to show efficacy in patients with moderate to severe RA, suggesting that inhibition of IL-17, one of the many cytokines produced by Th17 cells, may not be efficacious in RA (111, 112). Additionally, in Crohn's disease, trials of secukinum$\mathrm{ab}$ and brodalumab failed to show efficacy and even exacerbated disease activity in some patients (113). This is in line with evidence that IL-17A may be protective in murine colitis models (114). Thus, the clinical utility of targeting IL-17 depends greatly on the disease and highlights that IL-17 can play both pathogenic and protective roles in autoimmunity.
Agents targeting IL-23 are also in development. Monoclonal antibodies targeting IL-23/IL-12p40 have shown clinical efficacy for both psoriasis and Crohn's disease. Ustekinumab, an antibody to the shared IL-12/23p40 subunit, dramatically improves psoriasis, performing better than etanercept (115-117). Intriguingly, ustekinumab showed benefit in patients with Crohn's disease, including those with TNF-antagonist refractory disease (118). This is stark contrast to the results seen with inhibition of IL-17 and suggests that IL-23 and IL-17 may play distinct roles in the pathobiology of Crohn's disease.

\section{Conclusions}

Interactions between diet, the microbiota, and intestinal immune cells can markedly alter both systemic immune function and host metabolism, and this appears to be largely mediated by cytokines, particularly those in the IL-23/IL-17 axis. In this regard, intestinal ILC3s and Th cells demonstrate reduced production of IL-22 in response to IL-23 in some mouse models of obesity, leading to altered intestinal barrier function (119). Remarkably, administration of exogenous IL-22 not only rescued barrier function and reduced evidence of inflammation, but also led to improvement in glycemic control and dyslipidemia, highlighting the importance of interactions between the immune system, the microbiota, and host metabolic pathways in maintaining homeostasis. It is also important to note that the development of atherosclerosis is accelerated in several systemic human autoimmune diseases, particularly RA (120). It is thus intriguing to speculate that there are similar pathobiologic processes in play for both autoimmunity and metabolic diseases with an inflammatory component, such as type 2 diabetes and atherosclerosis.

While the past several decades have seen marked changes in diet, it is also likely that improvements in hygiene, the development of antibiotics, and widespread vaccination have resulted in significant changes in the intestinal microbiota $(121,122)$. This raises the possibility that altered regulation of cytokines as a consequence of changes in diet, metabolism, and commensal microbes, particularly in the intestinal microenvironment, may contribute to the increased incidence of autoimmune diseases, especially those involving the IL-23/IL-17 axis (Figure 3). Further investigation of the manner in which these complex and interrelated systems affect cytokine regulation represents a promising area of investigation to better understand the pathobiology of autoimmune disease and is already bearing fruit for identifying promising therapeutic targets to ease the human burden of these diseases.

\section{Acknowledgments}

The authors would like to acknowledge funding support from the NIH (P.R. Burkett and V.K. Kuchroo), the Deutsche Forschungsgemeinschaft (G. Meyer zu Horste), the National Multiple Sclerosis Society (G. Meyer zu Horste and V.K. Kuchroo), and the Crohn's and Colitis Foundation of America (V.K. Kuchroo).

Address correspondence to: Vijay K. Kuchroo, Brigham and Women's Hospital and Harvard Medical School, 77 Avenue Louis Pasteur, HIM 785, Boston, Massachusetts 02115, USA. Phone: 617.525.5537; E-mail: vkuchroo@rics.bwh.harvard.edu. 
1. Koch-Henriksen N, Sorensen PS. The changing demographic pattern of multiple sclerosis epidemiology. Lancet Neurol. 2010;9(5):520-532.

2. Gillespie KM, et al. The rising incidence of childhood type 1 diabetes and reduced contribution of high-risk HLA haplotypes. Lancet. 2004;364(9446):1699-1700.

3. Molodecky NA, et al. Increasing incidence and prevalence of the inflammatory bowel diseases with time, based on systematic review. Gastroenterology. 2012;142(1):46-54.

4. Mosmann TR, Cherwinski H, Bond MW, Giedlin MA, Coffman RL. Two types of murine helper T cell clone. I. Definition according to profiles of lymphokine activities and secreted proteins. JImmunol. 1986;136(7):2348-2357.

5. Yamane H, Paul WE. Cytokines of the gamma(c) family control CD $4+\mathrm{T}$ cell differentiation and function. Nat Immunol. 2012;13(11):1037-1044.

6. Croxford AL, Mair F, Becher B. IL-23: one cytokine in control of autoimmunity. Eur J Immunol. 2012;42(9):2263-2273.

7. Gaffen SL, Jain R, Garg AV, Cua DJ. The IL23-IL-17 immune axis: from mechanisms to therapeutic testing. Nat Rev Immunol. 2014;14(9):585-600.

8. Ferber IA, et al. Mice with a disrupted IFN- $\gamma$ gene are susceptible to the induction of experimental autoimmune encephalomyelitis (EAE). J Immunol.1996;156(1):5-7.

9. Matthys P, Vermeire K, Mitera T, Heremans H, Huang S, Billiau A. Anti-IL-12 antibody prevents the development and progression of collageninduced arthritis in IFN- $\gamma$ receptor-deficient mice. Eur Immunol. 1998;28(7):2143-2151.

10. Bettelli E, et al. Loss of T-bet, but not STAT1, prevents the development of experimental autoimmune encephalomyelitis. JExp Med. 2004;200(1):79-87.

11. Oppmann B, et al. Novel p19 protein engages IL$12 \mathrm{p} 40$ to form a cytokine, IL-23, with biological activities similar as well as distinct from IL-12. Immunity. 2000;13(5):715-725.

12. Cua DJ, et al. Interleukin-23 rather than interleukin-12 is the critical cytokine for autoimmune inflammation of the brain. Nature. 2003;421(6924):744-748.

13. Langrish CL, et al. IL-23 drives a pathogenic $T$ cell population that induces autoimmune inflammation. J Exp Med. 2005;201(2):233-240.

14. Aggarwal S, Ghilardi N, Xie MH, de Sauvage FJ, Gurney AL. Interleukin-23 promotes a distinct CD4 $\mathrm{T}$ cell activation state characterized by the production of interleukin-17. J Biol Chem. 2003;278(3):1910-1914.

15. Yen $\mathrm{D}$, et al. IL-23 is essential for T cell-mediated colitis and promotes inflammation via IL-17 and IL-6. J Clin Invest. 2006;116(5):1310-1316.

16. Uhlig $\mathrm{HH}$, et al. Differential activity of IL-12 and IL-23 in mucosal and systemic innate immune pathology. Immunity. 2006;25(2):309-318.

17. Ahern PP, et al. Interleukin-23 drives intestinal inflammation through direct activity on T cells. Immunity. 2010;33(2):279-288.

18. Parham C, et al. A receptor for the heterodimeric cytokine IL-23 is composed of IL-12R $\beta 1$ and a novel cytokine receptor subunit, IL-23R. J Immunol. 2002;168(11):5699-5708.
19. Bettelli E, et al. Reciprocal developmental pathways for the generation of pathogenic effector TH17 and regulatory T cells. Nature. 2006;441(7090):235-238.

20. Veldhoen M, Hocking RJ, Atkins CJ, Locksley $\mathrm{RM}$, Stockinger B. TGF $\beta$ in the context of an inflammatory cytokine milieu supports de novo differentiation of IL-17-producing T cells. Immunity. 2006;24(2):179-189.

21. Korn T, et al. IL-21 initiates an alternative pathway to induce proinflammatory $\mathrm{T}(\mathrm{H}) 17$ cells. Nature. 2007;448(7152):484-487.

22. Nurieva R, et al. Essential autocrine regulation by IL-21 in the generation of inflammatory T cells. Nature. 2007;448(7152):480-483.

23. Zhou L, et al. IL-6 programs T(H)-17 cell differentiation by promoting sequential engagement of the IL-21 and IL-23 pathways. Nat Immunol. 2007;8(9):967-974.

24. Ivanov, et al. The orphan nuclear receptor RORgammat directs the differentiation program of proinflammatory IL-17+ T helper cells. Cell. 2006;126(6):1121-1133.

25. Sonderegger I, Kisielow J, Meier R, King C, Kopf M. IL-21 and IL-21R are not required for development of Th17 cells and autoimmunity in vivo. Eur JImmunol. 2008;38(7):1833-1838.

26. Coquet JM, Chakravarti S, Smyth MJ, Godfrey DI. Cutting edge: IL-21 is not essential for Th17 differentiation or experimental autoimmune encephalomyelitis. JImmunol. 2008;180(11):7097-7101.

27. Sutherland AP, et al. Interleukin-21 is required for the development of type 1 diabetes in NOD mice. Diabetes. 2009;58(5):1144-1155.

28. Datta S, Sarvetnick NE. IL-21 limits peripheral lymphocyte numbers through $\mathrm{T}$ cell homeostatic mechanisms. PLoS One. 2008;3(9):e3118.

29. Lan Y, Luo B, Wang JL, Jiang YW, Wei YS. The association of interleukin-21 polymorphisms with interleukin-21 serum levels and risk of systemic lupus erythematosus. Gene. 2014;538(1):94-98.

30. Webb R, et al. A polymorphism within IL21R confers risk for systemic lupus erythematosus. Arthritis Rheum. 2009;60(8):2402-2407.

31. Nohra R, et al. RGMA and IL21R show association with experimental inflammation and multiple sclerosis. Genes Immun. 2010;11(4):279-293.

32. Marquez A, et al. Novel association of the interleukin 2-interleukin 21 region with inflammatory bowel disease. Am J Gastroenterol. 2009;104(8):1968-1975.

33. Todd JA, et al. Robust associations of four new chromosome regions from genome-wide analyses of type 1 diabetes. Nat Genet. 2007;39(7):857-864.

34. Hollis-Moffatt JE, et al. Only one independent genetic association with rheumatoid arthritis within the KIAA1109-TENR-IL2-IL21 locus in Caucasian sample sets: confirmation of association of rs 6822844 with rheumatoid arthritis at a genome-wide level of significance. Arthritis Res Ther. 2010;12(3):R116.

35. McGeachy MJ, et al. TGF-beta and IL-6 drive the production of IL-17 and IL-10 by T cells and restrain T(H)-17 cell-mediated pathology. Nat Immunol. 2007;8(12):1390-1397.

36. McGeachy MJ, et al. The interleukin 23 receptor is essential for the terminal differentiation of interleukin 17-producing effector $\mathrm{T}$ helper cells in vivo. Nat Immunol. 2009;10(3):314-324.

37. Manel N, Unutmaz D, Littman DR. The differentiation of human $\mathrm{T}(\mathrm{H})-17$ cells requires transforming growth factor- $\beta$ and induction of the nuclear receptor ROR $\gamma$ t. Nat Immunol. 2008;9(6):641-649.

38. Acosta-Rodriguez EV, Napolitani G, Lanzavecchia A, Sallusto F. Interleukins $1 \beta$ and 6 but not transforming growth factor- $\beta$ are essential for the differentiation of interleukin 17-producing human Thelper cells. Nat Immunol. 2007;8(9):942-949.

39. Volpe $\mathrm{E}$, et al. A critical function for transforming growth factor- $\beta$, interleukin 23 and proinflammatory cytokines in driving and modulating human T(H)-17 responses. Nat Immunol. 2008;9(6):650-657.

40. Zielinski CE, et al. Pathogen-induced human TH17 cells produce IFN- $\gamma$ or IL-10 and are regulated by IL-1ß. Nature. 2012;484(7395):514-518.

41. Lee $\mathrm{Y}$, et al. Induction and molecular signature of pathogenic TH17 cells. Nat Immunol. 2012;13(10):991-999.

42. Ghoreschi K, et al. Generation of pathogenic $\mathrm{T}(\mathrm{H}) 17$ cells in the absence of TGF- $\beta$ signalling. Nature. 2010;467(7318):967-971.

43. Hirota K, et al. Fate mapping of IL-17-producing T cells in inflammatory responses. Nat Immunol. 2011;12(3):255-263.

44. Yang Y, et al. T-bet is essential for encephalitogenicity of both Th1 and Th17 cells. JExp Med. 2009;206(7):1549-1564.

45. Annunziato F, et al. Phenotypic and functional features of human Th17 cells. J Exp Med. 2007;204(8):1849-1861.

46. Cosmi $\mathrm{L}$, et al. Identification of a novel subset of human circulating memory $\mathrm{CD} 4(+) \mathrm{T}$ cells that produce both IL-17A and IL-4. J Allergy Clin Immunol. 2010;125(1):222-230.

47. Wang YH, et al. A novel subset of CD4(+) T(H)2 memory/effector cells that produce inflammatory IL-17 cytokine and promote the exacerbation of chronic allergic asthma. J Exp Med. 2010;207(11):2479-2491.

48. Wang Y, et al. The transcription factors T-bet and Runx are required for the ontogeny of pathogenic interferon-gamma-producing T helper 17 cells. Immunity. 2014;40(3):355-366

49. Duhen R, Glatigny S, Arbelaez CA, Blair TC, Oukka M, Bettelli E. Cutting edge: the pathogenicity of IFN- $\gamma$-producing Th17 cells is independent of T-bet. JImmunol. 2013;190(9):4478-4482.

50. Yosef N, et al. Dynamic regulatory network controlling TH17 cell differentiation. Nature 2013;496(7446):461-468

51. Ciofani M, et al. A validated regulatory network for Th17 cell specification. Cell. 2012;151(2):289-303.

52. Sherlock JP, et al. IL-23 induces spondyloarthropathy by acting on $\mathrm{ROR}-\gamma \mathrm{t}+\mathrm{CD} 3^{+} \mathrm{CD} 4$ CD8 ${ }^{-}$entheseal resident T cells. Nat Med. 2012;18(7):1069-1076.

53. Molle C, et al. Tristetraprolin regulation of interleukin 23 mRNA stability prevents a spontaneous inflammatory disease. J Exp Med. 2013;210(9):1675-1684.

54. Wu C, et al. Induction of pathogenic TH17 cells 
by inducible salt-sensing kinase SGK1. Nature. 2013;496(7446):513-517.

55. Kleinewietfeld M, et al. Sodium chloride drives autoimmune disease by the induction of pathogenic TH17 cells. Nature. 2013;496(7446):518-522.

56. Codarri L, et al. RORgammat drives production of the cytokine GM-CSF in helper T cells, which is essential for the effector phase of autoimmune neuroinflammation. Nat Immunol. 2011;12(6):560-567.

57. El-Behi M, et al. The encephalitogenicity of $\mathrm{T}(\mathrm{H}) 17$ cells is dependent on IL-1- and IL-23-induced production of the cytokine GM-CSF. Nat Immunol. 2011;12(6):568-575.

58. Nair RP, et al. Genome-wide scan reveals association of psoriasis with IL-23 and NF- $\mathrm{KB}$ pathways. Nat Genet. 2009;41(2):199-204.

59. Wellcome Trust Case Control Consortium, et al. Association scan of 14,500 nonsynonymous SNPs in four diseases identifies autoimmunity variants. Nat Genet. 2007;39(11):1329-1337.

60. Australo-Anglo-American Spondyloarthritis Consortium (TASC), et al. Genome-wide association study of ankylosing spondylitis identifies non-MHC susceptibility loci. Nat Genet. 2010;42(2):123-127.

61. Duerr RH, et al. A genome-wide association study identifies IL23R as an inflammatory bowel disease gene. Science. 2006;314(5804):1461-1463.

62. Pidasheva S, et al. Functional studies on the IBD susceptibility gene IL23R implicate reduced receptor function in the protective genetic variant R381Q. PLoS One. 2011;6(10):e25038.

63. Tsunemi $Y$, et al. Interleukin-12 p40 gene (IL12B) 3 '-untranslated region polymorphism is associated with susceptibility to atopic dermatitis and psoriasis vulgaris. J Dermatol Sci. 2002;30(2):161-166.

64. Capon F, et al. Sequence variants in the genes for the interleukin-23 receptor (IL23R) and its ligand (IL12B) confer protection against psoriasis. Hum Genet. 2007;122(2):201-206.

65. Cargill M, et al. A large-scale genetic association study confirms IL12B and leads to the identification of IL23R as psoriasis-risk genes. Am J Hum Genet. 2007;80(2):273-290.

66. Fisher SA, et al. Genetic determinants of ulcerative colitis include the ECM1 locus and five loci implicated in Crohn's disease. Nat Genet. 2008;40(6):710-712.

67. Danoy P, et al. Association of variants at $1 \mathrm{q} 32$ and STAT3 with ankylosing spondylitis suggests genetic overlap with Crohn's disease. PLoS Genet. 2010;6(12):e1001195.

68. Ellinghaus E, et al. Genome-wide meta-analysis of psoriatic arthritis identifies susceptibility locus at REL. J Invest Dermatol. 2012;132(4):1133-1140.

69. Ma CS, et al. Deficiency of Th17 cells in hyper IgE syndrome due to mutations in STAT3. JExp Med. 2008;205(7):1551-1557.

70. Minegishi Y, et al. Dominant-negative mutations in the DNA-binding domain of STAT3 cause hyper-IgE syndrome. Nature. 2007;448(7157):1058-1062.

71. de Beaucoudrey L, et al. Mutations in STAT3 and IL12RB1 impair the development of human IL-17-producing T cells. J Exp Med. 2008;205(7):1543-1550.
72. Milner JD, et al. Impaired T(H)17 cell differentiation in subjects with autosomal dominant hyperIgE syndrome. Nature. 2008;452(7188):773-776.

73. Flanagan SE, et al. Activating germline mutations in STAT3 cause early-onset multi-organ autoimmune disease. Nat Genet. 2014;46(8):812-814.

74. Milner JD, et al. Early-onset lymphoproliferation autoimmunity caused by germline STAT 3 gain-offunction mutations. Blood. 2014;125(4):591-599.

75. Atarashi K, et al. ATP drives lamina propria $\mathrm{T}(\mathrm{H}) 17$ cell differentiation. Nature. 2008;455(7214):808-812.

76. Ivanov, et al. Specific microbiota direct the differentiation of IL-17-producing T-helper cells in the mucosa of the small intestine. Cell Host Microbe. 2008;4(4):337-349.

77. Gaboriau-Routhiau V, et al. The key role of segmented filamentous bacteria in the coordinated maturation of gut helper $\mathrm{T}$ cell responses. Immunity. 2009;31(4):677-689.

78. Klaasen HL, Koopman JP, Poelma FG, Beynen AC. Intestinal, segmented, filamentous bacteria. FEMS Microbiol Rev. 1992;8(3-4):165-180.

79. Ivanov, et al. Induction of intestinal Th17 cells by segmented filamentous bacteria. Cell. 2009;139(3):485-498.

80. Goto Y, et al. Segmented filamentous bacteria antigens presented by intestinal dendritic cells drive mucosal Th17 cell differentiation. Immunity. 2014;40(4):594-607.

81. Yang $\mathrm{Y}$, et al. Focused specificity of intestinal TH17 cells towards commensal bacterial antigens. Nature. 2014;510(7503):152-156

82. Lochner M, et al. Restricted microbiota and absence of cognate TCR antigen leads to an unbalanced generation of Th17 cells. J Immunol. 2011;186(3):1531-1537.

83. Geem D, Medina-Contreras O, McBride M, Newberry RD, Koni PA, Denning TL. Specific microbiota-induced intestinal Th17 differentiation requires MHC class II but not GALT and mesenteric lymph nodes. JImmunol. 2014;193(1):431-438.

84. Lecuyer E, et al. Segmented filamentous bacterium uses secondary and tertiary lymphoid tissues to induce gut IgA and specific T helper 17 cell responses. Immunity. 2014;40(4):608-620.

85. Stepankova R, et al. Segmented filamentous bacteria in a defined bacterial cocktail induce intestinal inflammation in SCID mice reconstituted with CD45RBhigh CD4+ T cells. Inflamm Bowel Dis. 2007;13(10):1202-1211.

86. Wu HJ, et al. Gut-residing segmented filamentous bacteria drive autoimmune arthritis via $\mathrm{T}$ helper 17 cells. Immunity. 2010;32(6):815-827.

87. Berer K, et al. Commensal microbiota and myelin autoantigen cooperate to trigger autoimmune demyelination. Nature. 2011;479(7374):538-541.

88. Lee YK, Menezes JS, Umesaki Y, Mazmanian SK. Proinflammatory T-cell responses to gut microbiota promote experimental autoimmune encephalomyelitis. Proc Natl Acad Sci U S A. 2011;108(suppl 1):4615-4622.

89. Kriegel MA, Sefik E, Hill JA, Wu HJ, Benoist C, Mathis D. Naturally transmitted segmented filamentous bacteria segregate with diabetes protection in nonobese diabetic mice. Proc Natl Acad Sci U S A. 2011;108(28):11548-11553.

90. Rakoff-Nahoum S, Paglino J, Eslami-Varzaneh
F, Edberg S, Medzhitov R. Recognition of commensal microflora by toll-like receptors is required for intestinal homeostasis. Cell. 2004;118(2):229-241.

91. Kusu T, et al. Ecto-nucleoside triphosphate diphosphohydrolase 7 controls Th17 cell responses through regulation of luminal ATP in the small intestine. J Immunol. 2013;190(2):774-783.

92. Hepworth MR, et al. Innate lymphoid cells regulate $\mathrm{CD}^{+}{ }^{+} \mathrm{T}$-cell responses to intestinal commensal bacteria. Nature. 2013;498(7452):113-117.

93. Shih VF, et al. Homeostatic IL-23 receptor signaling limits Th17 response through IL-22-mediated containment of commensal microbiota. Proc Natl Acad Sci U S A. 2014;111(38):13942-13947.

94. Qiu J, et al. Group 3 innate lymphoid cells inhibit T-cell-mediated intestinal inflammation through aryl hydrocarbon receptor signaling and regulation of microflora. Immunity. 2013;39(2):386-399.

95. Upadhyay V, et al. Lymphotoxin regulates commensal responses to enable diet-induced obesity. Nat Immunol. 2012;13(10):947-953.

96. Zenewicz LA, et al. IL-22 deficiency alters colonic microbiota to be transmissible and colitogenic. JImmunol. 2013;190(10):5306-5312.

97. Sonnenberg GF, et al. Innate lymphoid cells promote anatomical containment of lymphoid-resident commensal bacteria. Science. 2012;336(6086):1321-1325.

98. Gregor MF, Hotamisligil GS. Inflammatory mechanisms in obesity. Annu Rev Immunol. 2011;29:415-445.

99. Gruber L, et al. High fat diet accelerates pathogenesis of murine Crohn's disease-like ileitis independently of obesity. PLoS One. 2013;8(8):e71661.

100.Okada Y, et al. Trans fatty acids exacerbate dextran sodium sulphate-induced colitis by promoting the up-regulation of macrophagederived proinflammatory cytokines involved in Thelper 17 cell polarization. Clin Exp Immunol. 2013;174(3):459-471.

101. Monk JM, et al. Dietary n-3 polyunsaturated fatty acids (PUFA) decrease obesity-associated Th17 cell-mediated inflammation during colitis. PLoS One. 2012;7(11):e49739.

102.Monk JM, et al. Th17 cell accumulation is decreased during chronic experimental colitis by ( $\mathrm{n}-3)$ PUFA in Fat-1 mice. J Nutr. 2012;142(1):117-124.

103. De Caterina R. n-3 fatty acids in cardiovascular disease. N EnglJMed. 2011;364(25):2439-2450.

104.Ananthakrishnan AN, et al. Long-term intake of dietary fat and risk of ulcerative colitis and Crohn's disease. Gut. 2014;63(5):776-784.

105. Farez MF, Fiol MP, Gaitan MI, Quintana FJ, Correale J. Sodium intake is associated with increased disease activity in multiple sclerosis. J Neurol Neurosurg Psychiatry. 2014;86(1):26-31.

106.Sundström B, Johansson I, Rantapää-Dahlqvist S. Interaction between dietary sodium and smoking increases the risk for rheumatoid arthritis: results from a nested case-control study. Rheumatology (Oxford). 2015;54(3):487-493.

107. Langley RG, et al. Secukinumab in plaque psoriasis - results of two phase 3 trials. N EnglJMed. 2014;371(4):326-338.

108. Papp KA, et al. Efficacy and safety of secukinum$a b$ in the treatment of moderate-to-severe plaque 
psoriasis: a randomized, double-blind, placebocontrolled phase II dose-ranging study. Br J Dermatol. 2013;168(2):412-421.

109. Papp KA, et al. Brodalumab, an anti-interleukin17-receptor antibody for psoriasis. $\mathrm{N} \mathrm{EnglJ} \mathrm{Med.}$ 2012;366(13):1181-1189.

110. Mease PJ, et al. Brodalumab, an anti-IL17RA monoclonal antibody, in psoriatic arthritis. N Engl J Med. 2014;370(24):2295-2306.

111. Genovese MC, et al. Efficacy and safety of secukinumab in patients with rheumatoid arthritis: a phase II, dose-finding, double-blind, randomised, placebo controlled study. Ann Rheum Dis. 2013;72(6):863-869.

112. Martin DA, et al. A phase Ib multiple ascending dose study evaluating safety, pharmacokinetics, and early clinical response of brodalumab, a human anti-IL-17R antibody, in methotrexateresistant rheumatoid arthritis. Arthritis Res Ther.
2013;15(5):R164.

113. Hueber W, et al. Secukinumab, a human anti-IL17A monoclonal antibody, for moderate to severe Crohn's disease: unexpected results of a randomised, double-blind placebo-controlled trial. Gut. 2012;61(12):1693-1700.

114. O'Connor W Jr, et al. A protective function for interleukin 17A in T cell-mediated intestinal inflammation. Nat Immunol. 2009;10(6):603-609.

115. Leonardi CL, et al. Efficacy and safety of ustekinumab, a human interleukin-12/23 monoclonal antibody, in patients with psoriasis: 76-week results from a randomised, doubleblind, placebo-controlled trial (PHOENIX 1). Lancet. 2008;371(9625):1665-1674.

116. Papp KA, et al. Efficacy and safety of ustekinum$a b$, a human interleukin-12/23 monoclonal antibody, in patients with psoriasis: 52-week results from a randomised, double-blind, placebo-controlled trial (PHOENIX 2). Lancet. 2008;371(9625):1675-1684.

117. Griffiths CE, et al. Comparison of ustekinumab and etanercept for moderate-to-severe psoriasis. N EnglJMed. 2010;362(2):118-128.

118. Sandborn WJ, et al. Ustekinumab induction and maintenance therapy in refractory Crohn's disease. N EnglJMed. 2012;367(16):1519-1528.

119. Wang X, et al. Interleukin-22 alleviates metabolic disorders restores mucosal immunity in diabetes. Nature. 2014;514(7521):237-241.

120. Shoenfeld Y, et al. Accelerated atherosclerosis in autoimmune rheumatic diseases. Circulation. 2005;112(21):3337-3347.

121. Blaser MJ. Who are we? Indigenous microbes and the ecology of human diseases. EMBO Rep. 2006;7(10):956-960.

122. Cox LM, Blaser MJ. Pathways in microbe-induced obesity. Cell Metab. 2013;17(6):883-894. 